\title{
BRAIN ACTIVITY DURING READING
}

PET was used to study the functional anatomy of reading in the intact brain of subjects examined at the Hammersmith Hospital, London, and other centers. The type of reading task and the exposure duration of the word stimuli were variables that influenced the patterns of brain activity. Three tasks were applied as follows: reading aloud, reading silently, and distinguishing words and pseudowords in a lexical decision task. Reading aloud and reading silently produced activity in the left posterior temporal lobe. Lexical decision involved the left inferior and middle frontal cortices and the supplementary motor area. Brain activity was greater for short exposure durations than for long durations. (Price CJ et al. Brain activity during reading. The effects of exposure duration and task. Brain Dec 1994;117:1255-1269). (Respond: Dr Cathy Price, MRC Cyclotron Unit, Hammersmith Hospital, Ducane Road, London W12 OHS, UK).

COMMENT. Small variations in experimental design may influence brain activity as measured by PET, and the association of specific reading tasks with discrete anatomical areas must be interpreted with caution. The authors stress that the aim of their study was to determine reasons for inconsistencies in previous reports.

\section{BEHAVIOR DISORDERS AND NOONAN'S SYNDROME}

The behavioral phenotype and psychiatric symptoms of 21 children with Noonan's syndrome are reported from the Departments of Child and Adolescent Psychiatry, and Clinical Genetics, Royal Manchester Children's Hospital, UK. Ages ranged from 2 to 16 years (median, 9 years). Non-behavioral abnormalities included pregnancy complications (52\%), short stature (71\%), visual problems (57\%), and language impairment (43\%). Behavioral abnormalities in $>50 \%$ of the group included clumsiness $(71 \%)$, faddy eating habits (67\%), fidgeting (62\%), stubbornness (62\%), echolalia (52\%), euphoria (52\%), and irritability (52\%). On the Child Behavior Checklist for parent reported psychiatric abnormalities, only social relationships and attention span were disturbed, and these are non-specific. A behavioral profile for Noonan's syndrome is suggested by these findings but compared to sibling controls, the abnormalities were not statistically significant. (Wood A et al. Behavioral aspects and psychiatric findings in Noonan's syndrome. Arch Dis Child Feb 1995;72:153-155). (Respond: Dr A Wood, Department of Child and Adolescent Psychiatry, Royal Manchester Children's Hospital, Hospital Road, Pendlebury, Manchester M27 4HA, UK).

COMMENT. Noonan's syndrome is a genetic disorder characterized by pulmonary stenosis, facial abnormalities, short stature, and learning difficulties. Few studies have emphasized behavioral abnormalities and a behavioral phenotype has not been delineated. The above behavioral characteristics may be helpful in diagnosis, counselling, and treatment. The association of Noonan's syndrome and neurofibromatosis has been described. (Listernick, Charrow, 1990; see Progress in Ped Neur II, 1994, p377).

\section{ATLANTOAXIAL INSTABILITY IN DOWN'S SYNDROME}

The radiographs and clinical evaluations of 90 children with Down's syndrome were reassessed after an interval of 5 years in a study of atlantoaxial instability (AAI) at the Derbyshire Children's Hospital and Infirmary, Derby, 